\title{
Substation Circuit Breaker SCADA Cut-Over
}

\author{
Nathan Stokes
}

April 2018

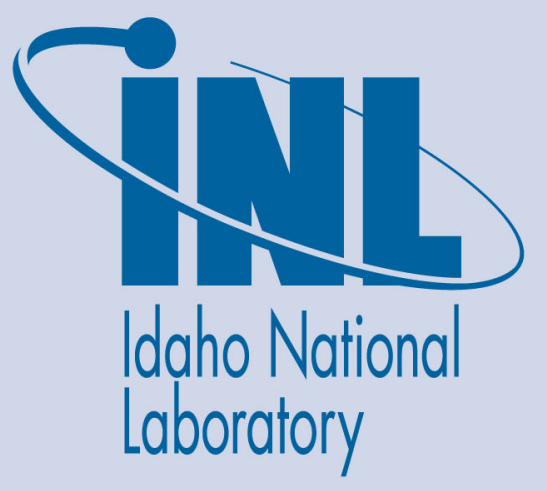

The INL is a U.S. Department of Energy National Laboratory operated by Battelle Energy Alliance 


\title{
Substation Circuit Breaker SCADA Cut-Over
}

\author{
Nathan Stokes
}

April 2018

Idaho National Laboratory Idaho Falls, Idaho 83415

http://www.inl.gov

Prepared for the U.S. Department of Energy Office of Nuclear Energy Under DOE Idaho Operations Office

Contract DE-AC07-05ID14517 
Nathan Stokes

Supervisory Control and Critical Infrastructure Systems, ECE 544

Dr Brian K. Johnson

Dr Frederick Sheldon

April 2, 2018

\section{Substation Circuit Breaker SCADA Cut-Over}

The electric power industry has a unique set of issues related to the infrastructure put in place since the 1880s. As methods of generation, delivery and protection evolve, so must the equipment used by the utility. Furthermore, the equipment in use has a finite life and must be replaced over time. The substation at which this project takes place was originally built in the 1950s, though most of the equipment had already gone through several updates. The issue presented was upgrading the Supervisory Control and Data Acquisition (SCADA) system for all the devices connected to the last SCADA system installed a few decades ago. Though the SCADA system being replaced was connected to substation transformers, breakers, disconnects, relays and other devices, the scope of this report will focus on the cut-over of a single gas circuit breaker. After connecting the circuit breaker to the new SCADA, a system and operability (SO) test would need to be designed and carried out to validate the proper function of the device before returning it to service. This report will follow the design approach, execution, testing, and troubleshooting for the substation circuit breaker SCADA cut-over.

Control for this breaker is available locally, in the substation, or remotely at the Power Dispatcher's computer console, referred to as a Human-Machine Interface or HMI. Bringing control for this breaker to the new SCADA and HMI would necessitate the temporary loss of remote control and taking the device out of service for the transition period. However, one requirement for this upgrade was that power was to remain uninterrupted; posing a complication. This was resolved by reconfiguring which devices fed the substation's switchgear, allowing this circuit breaker, and associated disconnects and transformer, to be taken offline 
without interrupting power to the customer. In order to modify the cables from the old Remote Terminal Unit (RTU) to the new RTU, the control circuit responsible for the breaker operation needed to be de-energized. This would ensure that the Linemen wouldn't be removing live conductors, but would also prevent an electrical short unintentionally tripping the circuit breaker. To accomplish this, the Linemen locked and tagged a breaker in a DC power panel in the open position. By placing a physical lock on the DC breaker, the Linemen maintain a safe work environment as someone would be unable to accidentally closing the breaker. Furthermore, the old RTU was also de-energized and the associated DC breaker was locked and tagged in the open position.

After the old RTU cabinet and the DC control circuit were de-energized, the next task was to remove the conductors that went from the old RTU cabinet to the gas circuit breaker. This circuit breaker's control cables did not go from the device to the old RTU cabinet directly but passed through a Field Terminal Panel (FTP). This allowed for half of the control cables, from the terminals inside the FTP back to the gas circuit breaker, to remain unchanged. The Linemen checked each of the identified cables for zero potential, verifying that the cables were in fact dead. Isolating the gas circuit breaker from the RTU allowed for power to be restored for the old SCADA system, to which the Power Dispatcher confirmed that status and control had been restored to the old HMI.

A similar method was used to maintain that the new RTU was and would remain de-energized while the Linemen worked on terminating cables. Conductors were then connected from the terminals in the FTP and landed at the corresponding terminals in the new RTU. Upon completion and having been double-checked, power was restored to the RTU and to the gas circuit breaker's control circuit. It was at this point that the Power Dispatcher noted that their new HMI was not able to reach the new RTU. Internal to the SCADA network, communication packets, in the form of pings, were successfully being sent and received from the HMI to the new network hub. This removed the possibility that the fiber lines were damaged or broken between the Dispatch center and the substation. Troubleshooting moved to the next variable. The networking hub had been installed recently in the substation where the SCADA cut-over was taking place and was only just connected to the new RTU via an Ethernet cable. After logging 
into the network hub and verifying the configuration, the port assigned to communicate with the new RTU was modified to allow communication from the newly connected device. With these settings applied, the RTU was now able to communicate with the Power Dispatcher's HMI.

The SCADA system is designed to be able to react to situations presented to it based on data acquired through the wiring that was completed earlier in this project. For instance, a gas circuit breaker needs to maintain a manufacturer specified pressure to function correctly. If the gas is allowed to drop to a low level, a mechanical contact internal to the pressure gauge completes an electrical circuit monitored, and sourced, by the new RTU. For this alarm, the Power Dispatcher is notified. If the breaker continues to lose gas pressure, a second contact in the pressure gauge completes a new electrical circuit. This time, however, the RTU processes the data and is programed to use other devices in conjunction with the circuit breaker to isolate the power grid from this gas circuit breaker before it cannot be relied upon to definitively break a circuit under tripping conditions. The RTU locks out the circuit breaker and also notifies the Dispatcher through the communication network, fiber lines and inevitably to the HMI.

It is not only a matter of procedure, but good engineering practice to validate each item modified in the system before returning anything back to service. The system and operability (SO) test is a thorough way to validate that devices affected by this project would function as intended for the next several decades. The SO test for this gas circuit breaker tested the following: Close Status, Open Status, Open Command, Close Command, Gas Alarm, and Spring Charge.

To test the Open and Close Statuses, the following SO test was performed. First, the clearances on the gas circuit breaker were released. This allows the breaker to be tested, however other devices were configured to isolate the circuit breaker from the electrical grid, preventing customers from being affected by these tests. With the gas circuit breaker in an open state, the dispatcher confirmed that their SCADA screen reflects the same status. Next, the Dispatcher sent a signal from their HMI to close the breaker. After the breaker closed, the Dispatcher confirms their HMI reflects the same status. This breaker is able to be operated remotely and locally, at the substation. Here, the Lineman manually opened and closed the circuit breaker while verifying the device and the SCADA status changed accordingly. Systematically, each scenario and circuit is 
tested to verify that the new RTU is able to operate as expected. One difference when testing certain alarms was that a jumper wire had been temporarily installed to simulate relays closing a contact based on conditions ascertained from data acquisition and processing. With that jumper installed, the Dispatcher was able to see alarms such as Gas Pressure and Spring Charge.

The issue presented of upgrading the SCADA system for any devices in or related to a substation is not a trivial one. Moving each wire requires careful planning, consulting diagrams, and skilled technicians and engineers. These conductors represent more than just some insulated copper; they represent a pathway for command signals, a conduit for data to be obtained and processed, and the added functionality to allow an operator or programming to interact with something like a circuit breaker. Through testing and troubleshooting this SCADA cut-over, one can have confidence that the device will operate as designed, with the help of the new RTU, for years to come. 